\title{
Isolation of an imported subgenotype B5 strain of human enterovirus $A 71$ in Chongqing City, China, 2014
}

\author{
Qian Yang ${ }^{1}$, Yong Zhang ${ }^{1}$, Dongmei Yan'1, Shuangli Zhu' ${ }^{1}$ Dongyan Wang ${ }^{1}$, Tianjiao Ji ${ }^{1}$, Wei Huang ${ }^{2}$, \\ Hongqiu $\mathrm{An}^{1}$ and Wenbo $\mathrm{Xu}^{1 *}$
}

\begin{abstract}
Background: Enterovirus A71 (EV-A71) is the main pathogen responsible for large outbreaks of hand, foot, and mouth disease (HFMD) in mainland China, and the dominant EV-A71 strains belong to subgenotype C4. To date, only one imported subgenotype B5 of EV-A71 has been reported in Xiamen City Fujian Province, 2009.

Results: Here, we report on another imported subgenotype B5 of EV-A71 isolated from a HFMD patient in Chongqing City in 2014 (strain CQ2014-86/CQ/CHN/2014, hereafter refer as CQ2014-86). The VP1 coding sequence and the whole genome sequence revealed that strain CQ2014-86 shares the high nucleotide identity with Vietnamese strains isolated in 2011-2013, suggesting that strain CQ2014-86 may have been imported from Vietnam. In the $5^{\prime} \mathrm{UTR}, \mathrm{P} 2$ and P3 regions, recombination events were found between strain CQ2014-86 and other EV-A, such as coxsackievirus A4 (CV-A4), CV-A5, CV-A14 and CV-A16.
\end{abstract}

Conclusions: This is the second report on importation of subgenotype B5 of EV-A71 in China, implying that we need to pay more attention to the importation of different subgenotypes of EV-A71.

Keywords: Human enterovirus A71, Subgenotype B5, Importation

\section{Background}

Human enterovirus A71 (EV-A71) is a neurotropic pathogen that can cause severe hand, foot, and mouth disease (HFMD) in children under 5 years old. It belongs to species EV-A (genus enterovirus, family picornaviridae, order Picornavirales). Following large scale outbreaks of HFMD in 2008 in mainland China, it has been categorized "C" group notifiable infectious diseases by the Ministry of Health of China [1-3]. Since then, China has built a HFMD laboratories network across the whole country, and this network is crucial for monitoring the prevalence of EV serotypes associated with HFMD patients, especially EV-A71. The molecular epidemiology of EV-A71 in mainland China reflects the pattern of circulation of subgenotype $\mathrm{C} 4$ viruses. In this study, we report on an imported

\footnotetext{
* Correspondence: wenbo_xu1@aliyun.com

'WHO WPRO Regional Polio Reference Laboratory and Key Laboratory of Medical Virology and Viral Disease, Ministry of Health, National Institute for Viral Disease Control and Prevention, Chinese Center for Disease Control and Prevention, Beijing 102206, People's Republic of China

Full list of author information is available at the end of the article
}

subgenotype B5 of EV-A71 isolated from a HFMD patient in Chongqing City, China, in 2014.

\section{Methods}

In total, 210 clinical samples were collected in Chongqing City in 2014. Viruses were isolated from the original clinical specimens by propagation in human rhabdomyosarcoma (RD) and human larynxarcinoma (HEp-2) cells by conventional methods. Viral RNA was extracted using QIAamp Viral RNA mini kit (QIAGEN, Germany). Realtime reverse transcription-polymerase chain reaction [4], molecular typing and phylogenetic analysis was performed as described previously [2]. And strain CQ2014-86 was identified as subgenotype B5 of EV-A71.

The viral RNA was converted to cDNA by a random priming strategy. The cDNA was amplified using primers according to the previous report [5], and a primer-walking strategy was used to close the gaps as necessary. PCR products were purified for sequencing using a QIAquick Gel Extraction Kit (QIAGEN), after 
which the amplicons were sequenced bidirectionally using fluorescent dideoxy-chain terminators and an ABI PRISM 3130 Genetic Analyzer (Applied Biosystems, Foster City, CA, USA). The 5'-segment sequence was determined using a 5'-Rapid Amplification of cDNA Ends Core Set (Takara Biomedicals, Dalian, China), according to the manufacturer's instructions.

The nucleotide sequences of EV-A71 strains was aligned using Bioedit sequence alignment editor software (version 5.0). Maximum likelihood (ML) trees were constructed using the best-fit Kimura 2-parameter + I model of nucleotide substitution in Mega software (version5.03) [6]. Similarity plot and bootscanning analyses were performed using the Simplot program (version 3.5.1; Stuart Ray, Johns Hopkins University, Baltimore, MD, USA).

\section{Results}

Not surprisingly, all EV-A71 strains belonged to subgenotype C4a except one strain, strain CQ2014-86/CQ/ $\mathrm{CHN} / 2014$ (hereafter refer as strain CQ2014-86), which was identified as subgenotype B5. Until now, only one whole genome sequence of subgenotype B5 from mainland China has been published (JN964686-Xiamen/ $\mathrm{FJ} / \mathrm{CHN} / 2009$ ) [5]. Here, we report the full-length genome sequence of a subgenotype B5 of EV-A71 (strain CQ2014-86) isolated in Chongqing City, China, during HFMD surveillance. The sequence of strain CQ2014-86 has been deposited in the GenBank database under the accession number KU647000. Strain CQ2014-86 was isolated from a throat swab specimen from a 4-year-old girl. The genome length of CQ2014-86 is 7412 nucleotides. Similarity between the only two strains that have full-length genomic sequences (Chongqing and Xiamen strains) isolated in mainland China is $95.75 \%$ at the nucleotide level and $98.49 \%$ at the amino acid level.

Phylogenetic analysis was conducted based on the entire VP1 coding region of Chongqing subgenotype B5 of EV-A71 sequence and other subgenotype B5 of EV-A71 sequences from the GenBank database (68 strains) (Fig. 1a). In addition, a phylogenetic tree based on the complete genome sequence of subgenotype B5 of EVA71 was also constructed (Fig. 1b).

CQ2014-86 shares the highest nucleotide identity with the Vietnamese strains. The nucleotide identities among

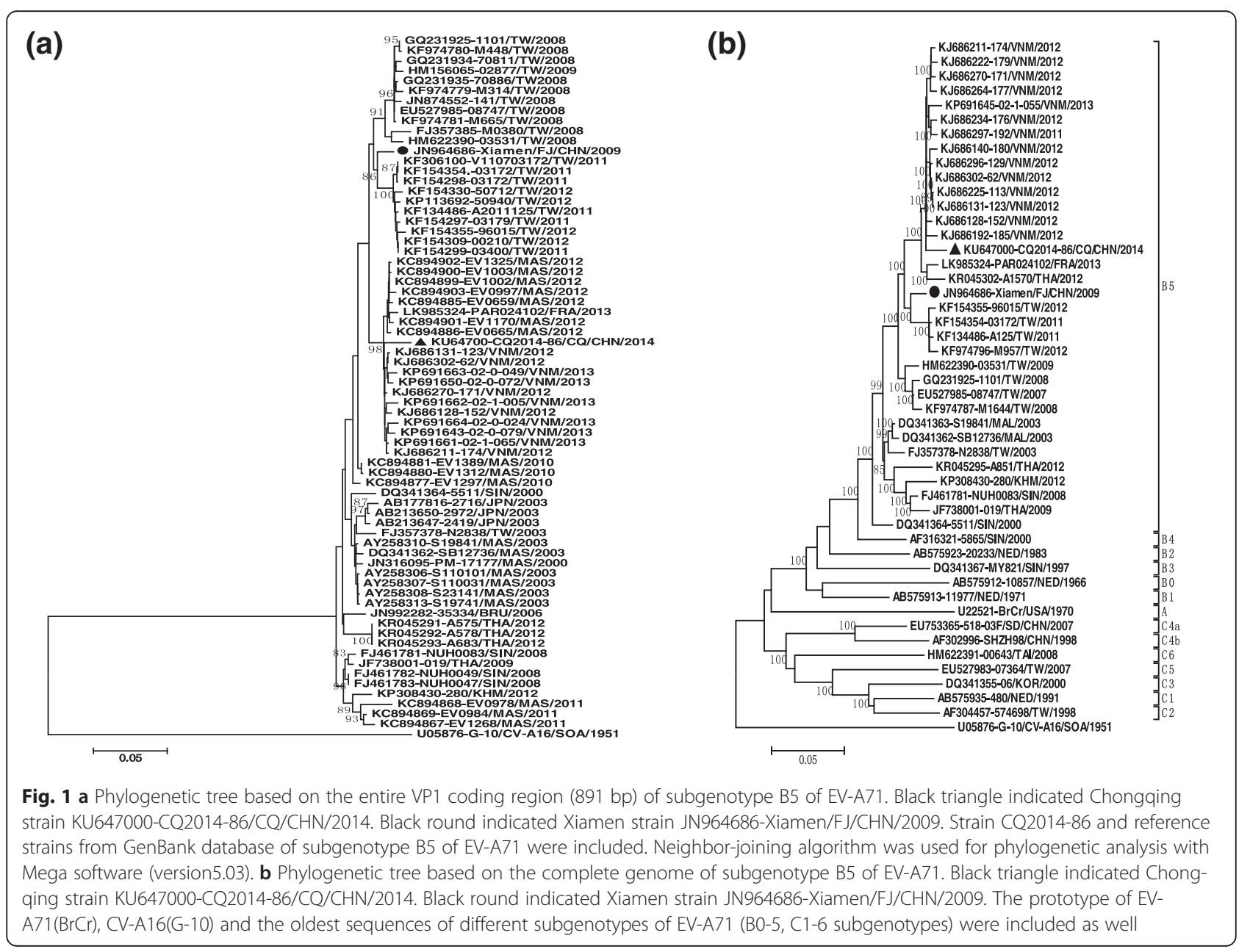




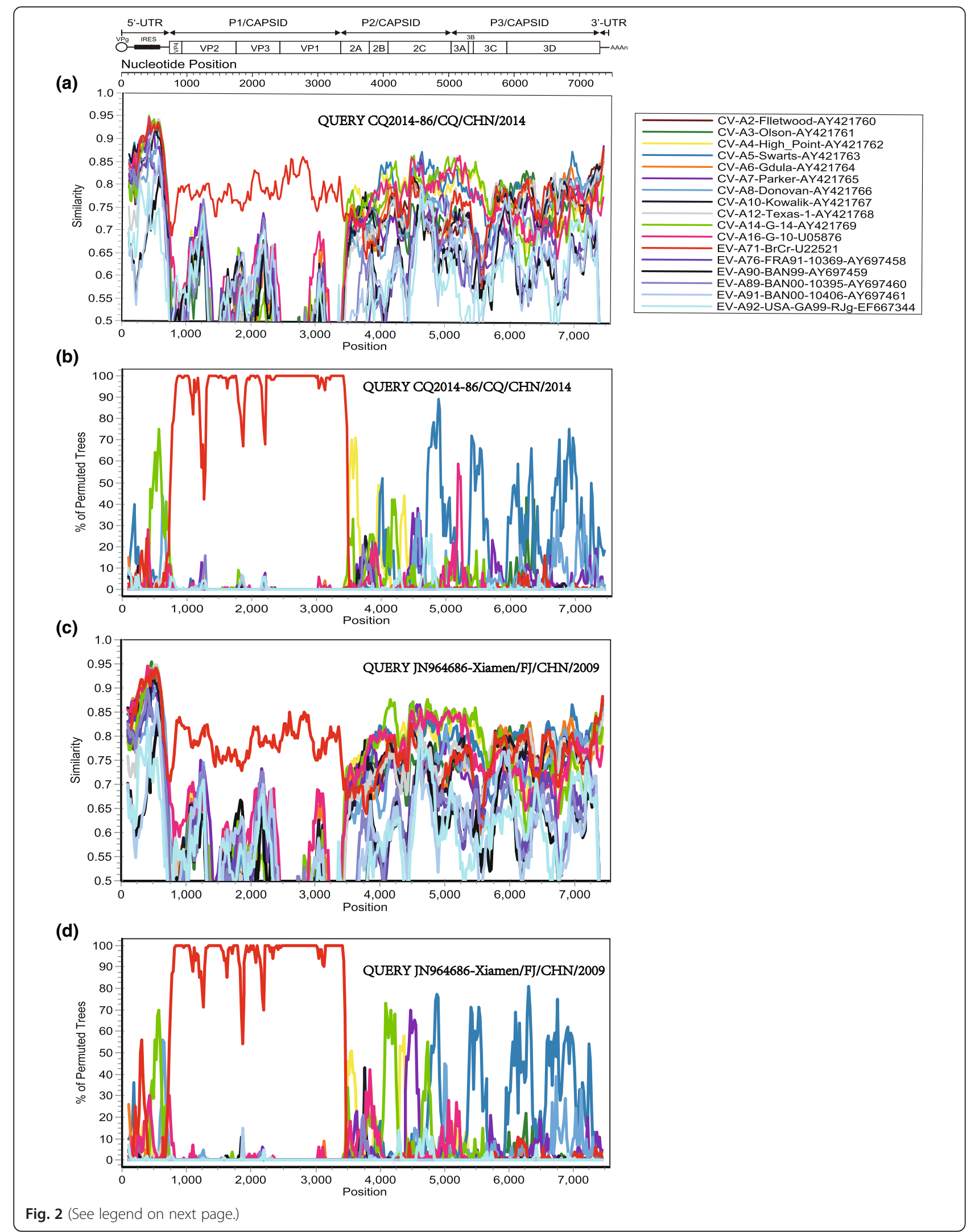


(See figure on previous page.)

Fig. 2 Similarity plot and bootscanning analyses of the whole genome of the EV-A strains. a Similarity plot and $\mathbf{b}$ bootscanning analysis. A sliding window of 200 nucleotides was used, moving in 20 nucleotide steps. The Chongqing strain CQ2014-86/CQ/CHN/2014 was used as the query sequence. c Similarity plot and $\mathbf{d}$ bootscanning analysis. A sliding window of 200 nucleotides was used, moving in 20 nucleotide steps. The Chongqing strain JN964686-Xiamen/FJ/CHN/2009 was used as the query sequence

them ranged from 97.5 to $99.9 \%$. The high similarity indicated that the strains had the same origin. Therefore, it is assumed that the virus was imported from neighboring countries and regions, possibly from Vietnam. Furthermore, the virus isolated from Xiamen City clustered with the strains from Taiwan, and the nucleotide identities between them ranged from 95.9 to $99.6 \%$, hence, the Xiamen strain may originate from Taiwan.

The similarity plot and bootscanning analysis revealed that recombination exists between CQ2014-86 and other EV-A strains, such as CV-A4, CV-A5, CV-A14, and CVA16 in the 5'UTR, and in the P2 and P3 regions (Fig. 2a and $\mathrm{b})$. Also, the Xiamen strain has a similar recombinant pattern, thus reconfirming that recombination is a common phenomenon in enterovirus. (Fig. 2c and d).

\section{Discussion}

The earliest subgenotype B5 of EV-A71 was isolated in Sarawak, Malaysia in 2000, and then in Yamagata, Japan in 2003 [7, 8]. The subgenotype B5, which is spread widely throughout the world, especially in Southeast Asia, has caused several disease outbreaks in Japan [7], Vietnam [9], Thailand [10], Singapore [11], Malaysia [8], and Taiwan $(2008,2012)[12,13]$.

In China, most of EV-A71 belonged to subgenotype C4 $[1,2]$. Frequent international travel, however, may result in some other subgenotypes being imported. This is the second report on importation of the subgenotype B5 of EV-A71 following the first, which occurred 5 years previously in Xiamen City in mainland China. Therefore, monitoring of imported subgenotypes EV-A71 should be strengthened.

\section{Acknowledgements}

We would like to acknowledge the staffs of the national HFMD surveillance program in the Chongqing Center for Disease Control and Prevention for collecting specimens from patients in this study. This study was supported by the National Basic Research Program of China (973 Program): (Grant No. 2011CB504902) and Key Technologies Research and Development Program of National Ministry of Science (Grant No. 2013ZX10004-202).

\section{Authors' contributions}

YZ and WX conceived and designed the experiments. QY, YZ, DY, SZ, DW, TJ, $\mathrm{WH}$ and HA performed the experiments. QY and $\mathrm{YZ}$ analyzed the data. QY wrote the main manuscript text. All authors reviewed the manuscript.

\section{Competing interests}

The authors declare that they have no competing interests.

\section{Declarations}

This study did not involve human participants or human experiments. The only human materials used were throat swabs from national HFMD surveillance at the instigation of the Ministry of Health P. R. of China for public health purpose. Written informed consent for the use of the clinical samples was obtained from the parents of the patient involved in this study. This study was approved by the second session of the Ethics Review

Committee of the National Institute for Viral Disease Control and Prevention, Chinese Center for Disease Control and Prevention, and the methods were carried out in accordance with the approved guidelines.

\section{Author details}

${ }^{1}$ WHO WPRO Regional Polio Reference Laboratory and Key Laboratory of Medical Virology and Viral Disease, Ministry of Health, National Institute for Viral Disease Control and Prevention, Chinese Center for Disease Control and Prevention, Beijing 102206, People's Republic of China. ${ }^{2}$ Chongqing Center for Disease Control and Prevention, Chongqing 400042, People's Republic of China.

Received: 29 March 2016 Accepted: 22 June 2016

Published online: 29 June 2016

\section{References}

1. Tan X, Huang X, Zhu S, Chen H, Yu Q, Wang H, Huo X, Zhou J, Wu Y, Yan D et al. The persistent circulation of enterovirus 71 in People's Republic of China: causing emerging nationwide epidemics since 2008. PLoS One. 2011; 6:e25662.

2. Zhang Y, Tan X, Wang H, Yan D, Zhu S, Wang D, Ji F, Wang X, Gao Y, Chen $L$ et al. An outbreak of hand, foot, and mouth disease associated with subgenotype C4 of human enterovirus 71 in Shandong. China J Clin Virol. 2009;44:262-7.

3. Zhang Y, Zhu Z, Yang W, Ren J, Tan X, Wang Y, Mao N, Xu S, Zhu S, Cui A et al. An emerging recombinant human enterovirus 71 responsible for the 2008 outbreak of hand foot and mouth disease in Fuyang city of China. Virol J. 2010;7:94. doi:10.1186/1743-422X-7-94

4. Cui A, Xu C, Tan X, Zhang Y, Zhu Z, Mao N, Lu Y, Xu W. The development and application of the two real-time RT-PCR assays to detect the pathogen of HFMD. PLoS One. 2013;8:e61451.

5. Zhang Y, Tan X, Cui A, Mao N, Xu S, Zhu Z, Zhou J, Shi J, Zhao Y, Wang X et al. Complete genome analysis of the $C 4$ subgenotype strains of enterovirus 71: predominant recombination C4 viruses persistently circulating in China for 14 years. PLoS One. 2013;8:e56341.

6. Tamura K, Peterson D, Peterson N, Stecher G, Nei M, Kumar S. MEGA5: molecular evolutionary genetics analysis using maximum likelihood, evolutionary distance, and maximum parsimony methods. Mol Biol Evol. 2011;28:2731-9.

7. Mizuta K, Abiko C, Murata T, Matsuzaki Y, Itagaki T, Sanjoh K, Sakamoto M, Hongo S, Murayama S, Hayasaka K. Frequent importation of enterovirus 71 from surrounding countries into the local community of Yamagata, Japan, between 1998 and 2003. J Clin Microbiol. 2005;43:6171-5.

8. Tee KK, Lam TTY, Chan YF, Bible JM, Kamarulzaman A, Tong CYW, Takebe Y, Pybus OG. Evolutionary Genetics of Human Enterovirus 71: Origin, Population Dynamics, Natural Selection, and Seasonal Periodicity of the VP1 Gene. J Virol. 2010;84:3339-50.

9. Geoghegan JL, Tan LV, Kuhnert D, Halpin RA, Lin X, Simenauer A, Akopov A, Das SR, Stockwell TB, Shrivastava S et al. Phylodynamics of Enterovirus A71Associated Hand, Foot, and Mouth Disease in Viet Nam. J Virol. 2015;89: 8871-9.

10. Puenpa J, Theamboonlers A, Korkong S, Linsuwanon P, Thongmee C, Chatproedprai S, Poovorawan Y. Molecular characterization and complete genome analysis of human enterovirus 71 and coxsackievirus A16 from children with hand, foot and mouth disease in Thailand during 2008-2011. Arch Virol. 2011;156:2007-13.

11. Wu Y, Yeo A, Phoon MC, Tan EL, Poh CL, Quak SH, Chow VT. The largest outbreak of hand; foot and mouth disease in Singapore in 2008: the role of enterovirus 71 and coxsackievirus A strains. Int J Infect Dis. 2010;14:1076-81. 
12. Huang YP, Lin TL, Kuo CY, Lin MW, Yao CY, Liao HW, Hsu LC, Yang CF, Yang JY, Chen PJ, Wu HS. The circulation of subgenogroups B5 and C5 of enterovirus 71 in Taiwan from 2006 to 2007. Virus Res. 2008;137:206-12.

13. Huang YP, Lin TL, Lin TH, Wu HS. Antigenic and genetic diversity of human enterovirus 71 from 2009 to 2012. Taiwan PLoS One. 2013;8:e80942.

Submit your next manuscript to BioMed Central and we will help you at every step:

- We accept pre-submission inquiries

- Our selector tool helps you to find the most relevant journal

- We provide round the clock customer support

- Convenient online submission

- Thorough peer review

- Inclusion in PubMed and all major indexing services

- Maximum visibility for your research

Submit your manuscript at www.biomedcentral.com/submit 